\title{
A Comparison between Kidney Allograft Biopsies Performed by Nephrologists and Surgeons Versus Interventional Radiologists
}

Sandeep Aggarwal ${ }^{1}$, Waqas I. Siddiqui 2, 3 , Nauman Shahid ${ }^{4}$, Jaime Baynes ${ }^{5}$, Muhammad W. Khattak 6 , Irfan Ahmed ${ }^{7,5}$, Suganthi Soundararajan ${ }^{8}$, Ziauddin Ahmed ${ }^{5}$

1. Nephrology, Drexel University College of Medicine, Philadelphia, USA 2. Cardiology/Nephrology, Drexel University College of Medicine, Philadelphia, USA 3. Cardiology/Nephrology, Orange Park Medical Center, Orange Park, USA 4. Nephrology, Eastern Nephrology Associates, Greenville, USA 5. Nephrology, Hahnemann University Hospital, Drexel University, Philadelphia, USA 6. Nephrology, Unity Point Health Methodist, Illinois Kidney Disease and Hypertension Center, Peoria, USA 7. Nephrology, Arizona Kidney Disease \& Hypertension Center, Chandler, USA 8. Pathology, Hahnemann University Hospital, Drexel University, Philadelphia, USA

Corresponding author: Waqas J. Siddiqui, dr.waqas20@gmail.com

\section{Abstract}

Introduction: Renal biopsy is an integral part of clinical nephrology practice that helps in the diagnosis of various renal diseases. Across the globe, it is performed by nephrologists and/or surgeons under ultrasound guidance. Lately, this novel procedure has been performed more frequently by the interventional radiologist (IR) as compared to nephrologists and surgeons.

Methods: We completed a retrospective review of 378 consecutive renal biopsies performed at our university hospital in the city center of Philadelphia, Pennsylvania, between September 2008 and June 2011 for various indications. Baseline characteristics were comparable except systolic blood pressure (SBP), prothrombin time (PT), and international normalized ratio (INR) which was higher. Hemoglobin was lower in patients who underwent biopsy by the IR compared to those who were biopsied by nephrologists and/or surgeons.

Results: The primary outcome showed the average number of glomeruli obtained with each biopsy was significantly lower by nephrology or surgical teams, $9.09 \pm 5.17$ vs. $19.17 \pm 11.11$ obtained by the interventional radiology team, $p$-value $<0.0001$. The number of cores obtained with each biopsy was significantly lower by nephrologist or surgeon at the bedside, $1.57 \pm 1.05$ vs. $2.42 \pm 1.26$, $\mathrm{p}$-value $<0.0001$. The average number of attempts to obtain one core was $2.00 \pm 1.10$ vs. $2.60 \pm 1.17$ by nephrologist and surgeon vs. IR, respectively, p-value $<0.0001$.

Conclusion: Our study clearly shows the superior success of renal biopsy by the IR as compared to the nephrology and surgical teams. This calls for more robust training of nephrology fellows and surgery residents to obtain the renal biopsy to prevent the loss of this unique procedure skill by non-radiology clinicians.

Received 11/30/2019

Review began 12/02/2019

Review ended 12/03/2019

Published 12/06/2019

(c) Copyright 2019

Aggarwal et al. This is an open access article distributed under the terms of the Creative Commons Attribution License CC-BY 3.0., which permits unrestricted use, distribution, and reproduction in any medium, provided the original author and source are credited.
Categories: Radiology, Nephrology

Keywords: clinical nephrology, interventional radiology, surgery, renal biopsy

\section{Introduction}

Kidney biopsies are indicated to make the diagnosis in patients with various renal diseases, including acute or chronic renal failure, delayed graft function, unexplained hematuria, and proteinuria [1-3]. Several complications have been associated with the procedure, such as perinephric hematoma, gross hematuria, hospitalization, arterio-venous fistula (AVF), and bowel perforations [3-4]. Most of the renal procedures, including renal biopsies, are part of the core curriculum of the Nephrology fellowship all over the world [5-6] and in most centers around the United States (US) [5-7]. Recently, renal biopsies are increasingly being performed by interventional radiologists (IR) compared to the nephrologists in training and surgeons who use the bedside ultrasound-guided percutaneous technique [8-10]. This is due to the loss of follow-up care and lack of training skills by the nephrology fellows as compared to the IR [10]. This transition has occurred to limit the complications associated with the ultrasound-guided bedside technique and to increase the safety, diagnostic yield, and efficacy of the procedure.

The number of glomeruli and the average number of cores obtained during the allograft biopsy is a cornerstone to increase the yield of the diagnostic specimen [10-11]. The number of attempts that it takes to obtain the precise sample is also essential because it decreases the chance of complications associated with the procedure [10-11]. There have been limited studies done to date comparing the efficacy, safety, and yield of renal biopsy and the cost-effectiveness between the bedside technique performed by the nephrology fellows and surgeons compared to the biopsy performed by the interventional radiologist [10]. Thus to compare both techniques, we performed this retrospective analysis to elucidate the factual difference that 


\section{Cureus}

exists between the two methods and to identify if there is any significant superiority that lies between the two techniques.

\section{Materials And Methods}

We completed a retrospective review of 378 consecutive renal biopsies done at our university hospital in the city center of Philadelphia, Pennsylvania between September 2008 and June 2011. Three hundred and seventy-eight biopsies were performed for various indications including protocol biopsies, rejection, and delayed graft function (DGF) or other reasons. Two hundred and forty biopsies were performed at the bedside after attaining all aseptic measures under the grayscale ultrasound guidance without biopsy needle guide, either by the nephrology fellows, nephrology attendings, the surgical residents and surgery attendings. One hundred and thirty-eight biopsies were done by the IR team in the radiology department. Eighteen gauge needles were used for all biopsies. The baseline characteristics of the patients who underwent renal biopsies are summarized in Table 1 .

\begin{tabular}{|c|c|c|c|}
\hline & Bed Side & Interventional Radiology Suite & p-value \\
\hline n. & 240 & 138 & \\
\hline Age & $53.11 \pm 13.5$ & $52.4 \pm 13.13$ & 0.816 \\
\hline Males/Females & $156 / 84$ & $94 / 44$ & 0.537 \\
\hline African American & 134 & 82 & 0.5 \\
\hline SBP & $135.84 \pm 17.86$ & $143.91 \pm 24.73$ & 0.0165 \\
\hline DBP & $75.69 \pm 13.58$ & $76.18 \pm 15.99$ & 0.826 \\
\hline MAP & $93.11 \pm 23.8$ & $81.68 \pm 40.42$ & 0.053 \\
\hline Hemoglobin & $12.00 \pm 1.83$ & $10.55 \pm 2.02$ & 0.001 \\
\hline Platelets & $217.51 \pm 71.24$ & $211.45 \pm 74.77$ & 0.546 \\
\hline PT & $11.79 \pm 1.35$ & $12.36 \pm 1.58$ & 0.007 \\
\hline INR & $1.03 \pm 0.11$ & $1.07 \pm 0.13$ & 0.01 \\
\hline PTT & $32.10 \pm 4.60$ & $33.00 \pm 10.08$ & 0.466 \\
\hline
\end{tabular}

\section{TABLE 1: Baseline Characteristics}

n. = Number, SBP = Systolic Blood Pressure, DBP = Diastolic Blood Pressure, MAP = Mean Arterial Pressure, PT = Prothrombin Time, INR = International Normalized Ratio, PTT = Partial Thromboplastin Time.

Age, African American race, and gender were equally matched. Systolic blood pressure was significantly higher in the patients who underwent biopsy by the IR but there was no difference in the diastolic blood pressure or the mean arterial pressure of the patients. Prothrombin time (PT) and international normalized ratio (INR) was also significantly higher in patients who got the biopsy with IR compared to the biopsies done at the bedside. There was significantly lower hemoglobin ( $\mathrm{Hgb}$ ) among patients who underwent the biopsy in the radiology department compared to the patients who got the biopsy at the bedside. There was no difference in partial thromboplastin time (PTT) or the platelet count as seen in Table 1 .

\section{Primary outcome}

The primary outcomes that were assessed were 1) the average number of glomerulus obtained with each biopsy, 2) average number of cores obtained with each biopsy and 3) average number of attempts to obtain one core.

\section{Secondary outcome}

The secondary outcomes were mainly the anticipated complications of a renal biopsy and they were 1) the procedural complication of gross hematuria, 2) need of hospitalization for any reason after the procedure, 3) perinephric hematoma, 4) AVF formation, 5) bowel perforation, 6) need for surgical intervention, and 7) need for blood transfusion.

\section{Statistical method}




\section{Cureus}

IBM Statistical Package for the Social Sciences (SPSS Inc., Chicago, IL) was used to calculate the statistical significance by comparing the means of the individual outcome. Chi-square test was used and the value of < 0.05 was considered to be statistically significant.

\section{Results}

The primary outcome included the average number of glomerulus obtained with each biopsy was $9.09 \pm 5.17$ by the nephrologist or the surgical team in the bedside group compared to $19.17 \pm 11.11$ obtained by the IR in the radiology department, $\mathrm{p}$-value $<0.0001$. The number of cores obtained with each biopsy at the bedside or by IR was $1.57 \pm 1.05$ vs. $2.42 \pm 1.26$, respectively p-value $<0.0001$. The average number of attempts to obtain one core was $2.00 \pm 1.10$ vs. $2.60 \pm 1.17$ at the bedside and IR, respectively, $\mathrm{p}$-value $<0.0001$ (Table 2 ).

\begin{tabular}{|l|l|l|}
\hline & Biopsy by Nephrologist or Surgeon at bed-side & Biopsy by Interventional Radiologist \\
\hline n. & 240 & 138 \\
\hline Numbers of Glomerulus & $9.09 \pm 5.17$ & $19.17 \pm 11.11$ \\
\hline Numbers of Crores & $1.57 \pm 1.05$ & $2.42 \pm 1.26$ \\
\hline Attempts per core & $2.00 \pm 1.10$ & $2.60 \pm 1.17$ \\
\hline
\end{tabular}

\section{TABLE 2: Primary Outcomes - Allograft Biopsy Tissue Yield}

p-value calculated using chi-square test.

The secondary outcomes showed that the most common complications were gross hematuria in four cases in the bedside group vs. one case in the IR group, $p$-value $=0.4402$. Patients requiring hospitalizations for the complications of the biopsy were six in the bedside biopsy group vs. one in the IR group, $p$-value $=0.2177$. One case of perinephric hematoma was observed in each group, $p$-value $=0.691$. There were two cases of the arteriovenous fistula in the bedside group but none in the IR group. There were no events of bowel perforation, need for surgical intervention, or need for blood transfusion in either group (Table 3).

\begin{tabular}{|c|c|c|c|}
\hline & Biopsy by Nephrologist or Surgeon at bed side & Biopsy by Interventional Radiologist & p-value \\
\hline n. & 240 & 138 & \\
\hline Gross Hematuria n. (\%) & $4(1.66)$ & $1(0.72)$ & 0.4402 \\
\hline Hospitalization n. (\%) & $6(2.5)$ & $1(0.72)$ & 0.2177 \\
\hline Perinephric Hematoma n. & $1(0.41)$ & $1(0.72)$ & 0.691 \\
\hline Arterio-Venous Fistula n. (\%) & $2(0.83)$ & 0 & \\
\hline Bowel Perforation & None & None & \\
\hline Need for surgical intervention n. (\%) & None & None & \\
\hline Blood Transfusion $\mathrm{n}$. & None & None & \\
\hline $\begin{array}{l}\text { TABLE 3: Secondary Outc } \\
\text { p-value calculated using chi-square te }\end{array}$ & mes - Complications & & \\
\hline
\end{tabular}

\section{Discussion}

Although this study shows a fewer number of cores and fewer attempts per core with bedside biopsies, it should be noticed that this was a single-center study with limited sample size. Moreover, the sample size was not equally distributed, with a significantly higher number of patients in the bedside group. It should also be noted that systolic blood pressure (SBP) and INR were, on average, higher in the IR biopsy group, which theoretically predispose those patients to higher post-procedural complications. Hence, having relatively fewer IR related biopsy complications, as compared to bedside biopsies, points towards better techniques by experienced interventional radiologists. 
This study provides a platform and lays a foundation for future studies by other academic institutions where patient's safety, cost-effectiveness, and training the residents and fellows are valued and evaluated. The number of glomeruli and the average number of cores obtained during a procedure is considered a cornerstone to increasing the yield of the diagnostic specimen [10-11]. The number of attempts that it takes to obtain the precise specimen is also important because it decreases the chance of complications associated with the procedure [10-11]. There have been limited studies done to date to compare the efficacy, safety, and yield of renal biopsy along with the cost-effectiveness between the bedside technique. To implement the routine bedside biopsies safely and effectively, an institution like ours need to devote resources for handson fellows and faculty training. This can be achieved by a dedicated period of training for residents and fellows during their training to learn, practice, and be proficient in these procedures.

In summary, the bedside biopsies, if done in a correct patient population, can be safe, efficient, and costeffective. It can be a major component of fellow's or residents' training as well as a key factor in reducing the healthcare financial burden on society.

\section{Conclusions}

Renal biopsies have been part of the nephrology and surgical practice. With the recent advent of interventional techniques, more and more renal biopsies are being performed by the IRs. Thus making the future nephrologists less comfortable with bedside biopsies leading to an increased number of biopsies being performed by the IR. This leads to an increase in healthcare costs. A structured and dedicated time as part of the nephrology fellowship and surgical training will aide in providing skills for a safe and successful biopsy at the bedside. It can, in turn, minimize the interventional radiology referrals, healthcare costs, and delay patient care.

\section{Additional Information \\ Disclosures}

Human subjects: Consent was obtained by all participants in this study. Animal subjects: All authors have confirmed that this study did not involve animal subjects or tissue. Conflicts of interest: In compliance with the ICMJE uniform disclosure form, all authors declare the following: Payment/services info: All authors have declared that no financial support was received from any organization for the submitted work. Financial relationships: All authors have declared that they have no financial relationships at present or within the previous three years with any organizations that might have an interest in the submitted work. Other relationships: All authors have declared that there are no other relationships or activities that could appear to have influenced the submitted work.

\section{References}

1. Agarwal SK, Sethi S, Dinda AK: Basics of kidney biopsy: a nephrologist's perspective . Indian J Nephrol. 2013, 23:243-52. 10.4103/0971-4065.114462

2. Draman CR, Seman MR, Mohd Noor FS, Kelsom WM: Diagnostic yield of kidney biopsies performed in a suburban, satellite hospital. Saudi J Kidney Dis Transpl. 2013, 24:178-83. 10.4103/1319-2442.106365

3. Yaqub S, Ahmed N, Fatima U, Maqbool A, Ashif W, Hussain SA: Complication rate and diagnostic yield of percutaneous native kidney biopsies: a 10-year experience at a Tertiary Care Hospital in Pakistan. Saudi J Kidney Dis Transpl. 2017, 28:891-7.

4. Rasmussen LR, Loft M, Nielsen TK, Jensen MB, Hoyer S, Horlyck A, Graumann O: Short-term complications for percutaneous ultrasound-guided biopsy of renal masses in adult outpatients. Acta Radiol. 2018, 59:491496. 10.1177/0284185117720855

5. Yesudas SS, Georgy NK, Manickam S, Raheena A, Monai RC, Noble BA, Pillai A: Percutaneous real-time ultrasound-guided renal biopsy performed solely by nephrologists: a case series. Indian J Nephrol. 2010, 20:137-41. 10.4103/0971-4065.70844

6. Berns JS, O'Neill WC: Performance of procedures by nephrologists and nephrology fellows at U.S. nephrology training programs. Clin J Am Soc Nephrol. 2008, 3:941-7. 10.2215/CJN.00490108

7. Fulop T, Alemu B, Dossabhoy NR, et al.: Safety and efficacy of percutaneous renal biopsy by physicians-intraining in an academic teaching setting. South Med J. 2014, 107:520-5. 10.14423/SMJ.0000000000000148

8. Hergesell O, Felten H, Andrassy K, Kuhn K, Ritz E: Safety of ultrasound-guided percutaneous renal biopsyretrospective analysis of 1090 consecutive cases. Nephrol Dial Transplant. 1998, 13:975-7. 10.1093/ndt/13.4.975

9. Torres Munoz A, Valdez-Ortiz R, Gonzalez-Parra C, Espinoza-Davila E, Morales-Buenrostro LE, CorreaRotter R: Percutaneous renal biopsy of native kidneys: efficiency, safety and risk factors associated with major complications. Arch Med Sci. 2011, 7:823-31. 10.5114/aoms.2011.25557

10. Gupta RK, Balogun RA: Native renal biopsies: complications and glomerular yield between radiologists and nephrologists. J Nephrol. 2005, 18:553-8.

11. Kriegshauser JS, Patel MD, Young SW, Chen F, Eversman WG, Chang YH, Smith M: Factors contributing to the success of ultrasound-guided native renal biopsy. J Ultrasound Med. 2016, 35:381-7.

10.7863/ultra.15.05023 